\title{
Testing the Efficacy of Platform and Train Passenger Boarding, Alighting and Dispersal Through Innovative 3D Agent-Based Modelling Techniques
}

\author{
Selby Coxon ${ }^{1} \cdot$ Tom Chandler $^{2} \cdot$ Elliott Wilson $^{2}$
}

Received: 13 March 2015/Revised: 17 May 2015/Accepted: 26 May 2015/Published online: 2 September 2015

(C) The Author(s) 2015. This article is published with open access at Springerlink.com

\begin{abstract}
Suburban railways around the world are experiencing a rapid increase in patronage. Higher passenger densities, particularly during peak times of the day, have implications for train punctuality, crowding, accessibility and passenger comfort. Research indicates that the design of the train carriage and the impediments of platform furniture all have an influence on accessibility and passenger dispersal, with consequences for service punctuality and network capacity. Building new concepts in train and station design are expensive undertakings and carry with the investment a high level of risk. Computational simulation methods such as agent-based modelling (ABM) can mitigate this risk at much lower cost. Many contemporary ABM modellers represent passenger flow at a macroscale, often in a single plan view and with agents travelling at same speeds and represented crudely as dots on a flat plane. This paper discusses a body of work concerning the building of a boarding and alighting simulator at a more detailed scale where a deeper and richer experience of crowd behaviour has been modelled using 3D animated figures. The primary benefit of these methods of evaluation is that they take away the expense and lack of realism present in experiments with full-size mock-ups. The outcomes of this work have resulted in sophisticated imagery, underpinned by technical accuracy that provides a tool for
\end{abstract}

Selby Coxon

selby.coxon@monash.edu

1 Faculty of Art Design \& Architecture, Monash University, 900 Dandenong Road, Caulfield, Melbourne 3145, Australia

2 Faculty of Information Technology, Monash University, 900 Dandenong Road, Caulfield, Melbourne 3145, Australia

Editor: Baoming Han the development of station infrastructure, train carriage design with implications on timetabling and network planning.

Keywords Dwell time - Agent-based modelling · 3D

\section{Introduction}

Rail is an important contributor to the movement of people and goods in many of the world's large cities. Suburban, metro and subway systems are very efficient in terms of the number of people moved relative to land use. Rail is a popular means of transport and becoming more so as urban populations increase. In the latter part of the 19th century, when the London Underground opened, only $10 \%$ of the world's population lived in cities. Now in the early 21 st century, over $50 \%$ of the world's population live in a city [1]. In terms of transit use, $80 \%$ of the population of Tokyo uses the subway, making some 2930 million passenger journeys per year (2009 figure) (Ibid), the highest level of patronage anywhere in the world.

Trains are independent of congested road traffic conditions and therefore have the potential to be faster at delivering passengers into city centres. Automation and advances in signalling reduce the impediments to a smooth and timely rail system. The growth in city populations has fuelled increased rail patronage with the consequence that many train networks can struggle to be punctual. The most significant variable in the journey of a train is the time it will take paused at each station. This 'dwell' time is at the mercy of how long it takes passengers to board, alight and disperse within the train carriage or across the platform. At peak periods, dwell times can become extended as passengers jostle to board or alight. It is 
general practice that timetables have built-in 'recovery' time and attempt to predict extensions of dwell time during peak periods. However, with increased patronage, the predictability of dwell times becomes more difficult [4]. Delayed trains create a number of implications beyond poor punctuality, including the extension of headways (the time gap between services). This extension is especially onerous if the lines are shared with express services and freight trains. Extended dwell times reduce network capacity leading to less services tor's revenue and contributing to poor passenger perceptions of the mode.

Dwell time predictability is important in the creation of service timetables. To this end, operators subdivide the dwell time to better understand where problems lie. Current timetable orthodoxy determines dwell times by mathematical means. While there are variations to the formula, they all in essence treat boarding and alighting as a linear period of time multiplied by a coefficient representative of how much passengers have been slowed down by the circumstances of other passengers, width of the doors and if they are carrying belongings [5]. Accurate calculation of these dwell times will inform operators of the predicted capacity of the network and so drive timetables with some accuracy.

However, while building mathematical models might simplify determining dwell times as they may be, they also mask the intricate composition of the causes of extended dwells. Studies show [2] that there is a wide range of qualitative variables that impact upon passenger behaviour while boarding and alighting. These factors include the prevailing culture of the passengers, their age, relative athleticism, the gap between the platform and the train, the level of the occlusion at the door and their motivations once within the train to finding a seat. These human factor variables are difficult to be determined quantitatively, but they do relate strongly to the interface between the passenger and carriage. Figure 1 shows the points between predictable timing with where the unpredictable variation in dwell is located. Figure 2 encapsulates, as a flowchart, each of the 'factors' that affect the efficacy of the passenger and more late services, ultimately impacting upon an opera-

to board or alight from a train and by implication impact upon the dwell time variability of the service.

Extended dwell times imply difficulty in passenger boarding and alighting at anyone or more of the stages outlined above. With significant increases in patronage, particularly during peak time, crowding itself is the significant determinant of extended dwell times. While passengers may not be particularly aware of the wider implications of delays at the station during boarding and alighting, crowding (the cause of the delay) tends to have a greater impact especially upon the perception of comfort.

\section{Measurement and Evaluation Methods: The Role of Computational Modelling}

Historically, methods of determining boarding and alighting performance have been confined to the building of fullsize mock-ups and inviting a sample group of passengers to enter and alight from the interior and a platform structure. This method has varying degrees of success as a method of dwell time calibration. Documenting the experimental process through, for example, video aids the evaluation and decision making of the manufacturer. However, this method is time consuming and costly and to some extent flawed by the unreal nature of the setting [2].

Agent-Based Modelling seeks to direct digitally animated 'agents' by way of a series of algorithms originally derived empirically. ABM interactions exhibit the following two properties:

(1) The interactions are composed of individuals (agents) with a designated set of characteristics.

(2) The system in which these interactions take place exhibits emergent properties, that is, new properties arising from the interactions of the agents that cannot be deduced simply by aggregating the combined properties of the agents.

The primary benefit of these methods of evaluation is that they take away the expense and lack of realism present

Fig. 1 Linear diagram of dwell time structure

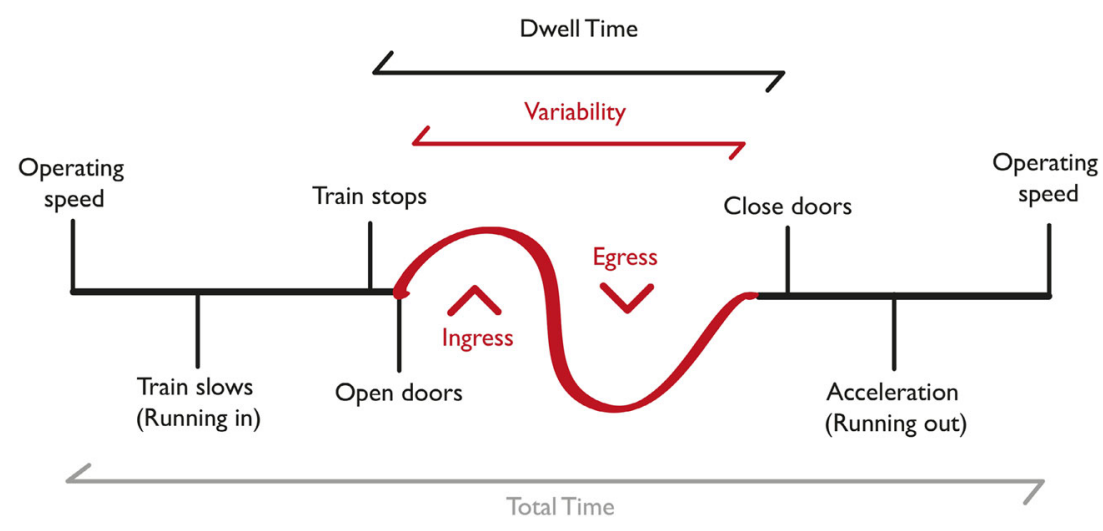




\section{Alighting}

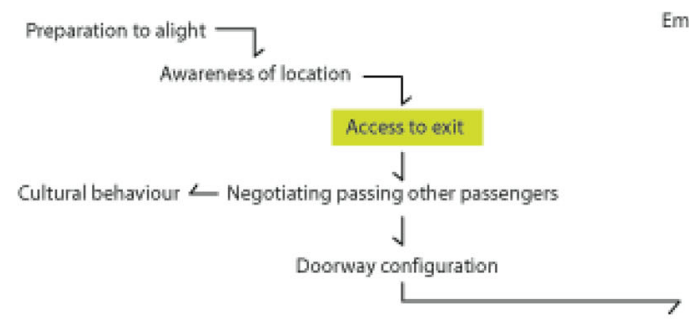

\section{Boarding}

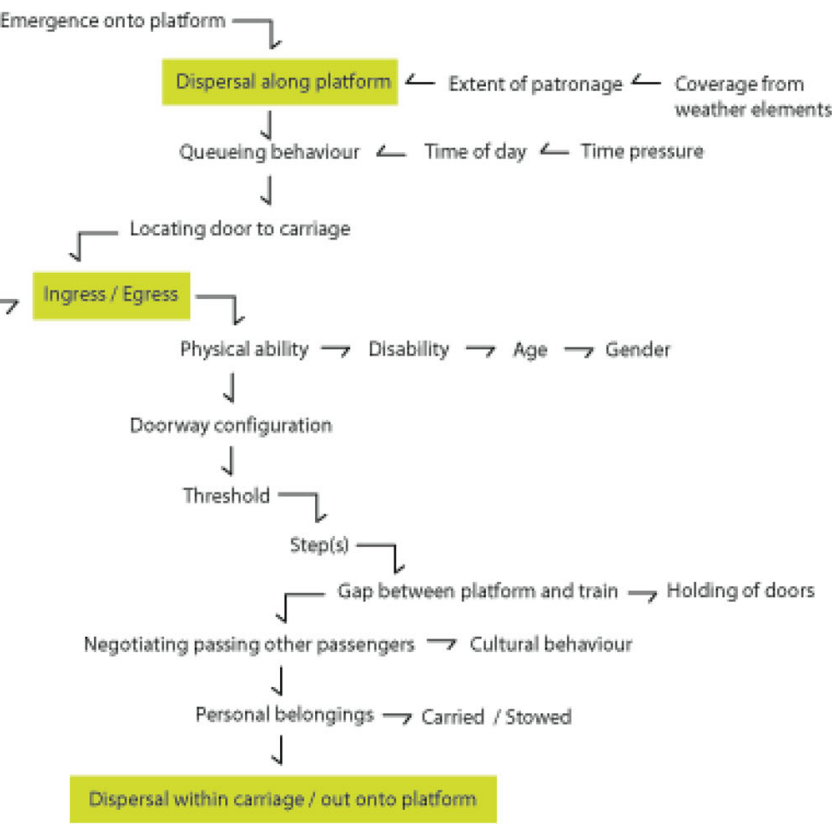

Fig. 2 Flow chart of boarding and alighting influences

in physical experiments with full-size mock-ups. In computer simulation, animated passengers are programmed to undertake simple tasks with directed goals, e.g. board and find the nearest free seat. This is done irrespective of any sense of urgency that might be present at a real boarding or lack of urgency at a static mock-up.

ABM begins with assumptions about the agents (passengers) and their interactions and then uses computer simulation to reveal the dynamic consequences of these assumptions. ABM researchers can investigate how large-scale effects arise from the micro-processes of interactions among many agents. Large-scale effects of interacting agents can be surprising because it can be hard to anticipate the full consequences of even simple forms of interaction. For problems such as determining the ebb and flow of large groups of train passengers where predicting the effects of individuals on each other is difficult, ABM techniques have great potential. What is difficult to determine is how accurate and representative the salient aspects of the agents are of the travelling public. In highly sophisticated simulations, it is possible to equip the agents with the ability to learn and develop over time. The key issue here is the extent to which the resulting outcomes are orderly within the environment where they have been placed.

\section{Developments in Computational ABM}

While the simulation of $3 \mathrm{D}$ environments is a long established practice in the computer-aided design and engineering disciplines, the inclusion of 3D human models with animated behaviours into crowd modelling spaces remains comparatively rare. Some of the reasons for the absence of human models can be attributed not only to the unique challenges involved in animating 3D figures, but also to the absence of animators and computer game researchers involved in the simulation design process. Recent advances in game engine technology and computer processing power have enabled human 'agents' to be realistically graphically depicted in simulations, walk cycles, idle cycles and a range of poses and animated behaviours that can enhance the interpretability of the simulation (Fig. 3).

\section{Creating the 3D Characters}

Fundamental to the authenticity of animated passengers is their walk cycle. For the 3D characters designed for the simulation discussed here, different animated walk cycles were designed to communicate whether an agent was male or female, young or old. Sped into a run, a walk cycle can also convey the urgency of the agent's objectives. When at rest, such as waiting for a train or as a passenger on board the animated characters function in an idle cycle, which is an animated loop of small and almost imperceptible movements that characters make when they are sitting or standing. Characters may, for example, shift their balance slowly from one foot to another or turn their head slightly in each direction to look about. The $3 \mathrm{D}$ characters in this research were modelled as Melbourne city commuters; they were realistic enough that their facial features could 


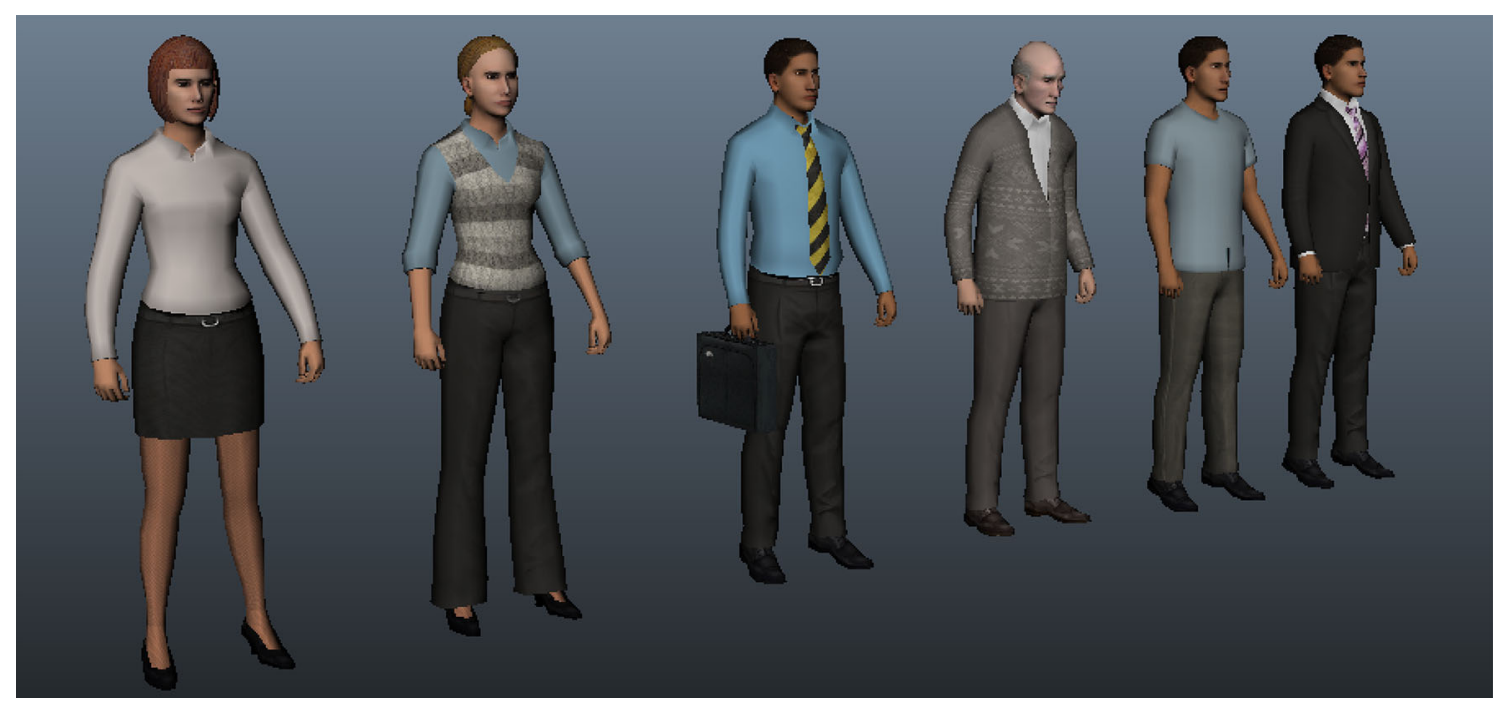

Fig. 3 Selection of 3D commuter characters: the visual and animated embodiments of the agents in the simulation. Created by Chandara Ung

be discerned and attire to indicate gender or replicate the dominant dress styles of commuters during peak periods. Their clothes were to some degree interchangeable and appropriate for Melbourne's temperate climate, though not for any particular season. The male and female figures ranged in age from high school students to retirees and accommodated a number of body shapes and ethnicities.

As the simulation was developed, the interplay of character typologies, attire and idle cycles presented challenges. For example, the sitting posture in the idle cycle differs for women and men reflecting their physiology. Creating a stance that could be perceived as relaxed and not tense required subtleties in the building of each iteration of character model. The building in of commonplace distractions such as the inspecting of mobile phones and the shifting of body weight all added to the appearance of a realistic environment (Fig. 4).

\section{Outline of Simulation Logic and Rendering}

The Unity game engine was created in 2005 by Unity Technologies. The software allows for the creation of interactive 3D content with minimal technical effort. Though mostly used in video game production, it has also become a popular visualisation and scientific research tool in a number of varied disciplines. In this project, the Authors used Unity to develop and test the efficacy of carriage interior seat and impediment arrangements. Unity has a pathfinding engine built-in which allows the author of the simulation to define which areas of the scene are "walkable" and the different kinds of "agents" that will move through the scene Fig. 5. Once these have been defined, the author can write code that will give the agent a goal position. The agent will then calculate the shortest path towards this goal through the "walkable" area thus avoiding issues such as walking through walls and other designated solid objects. The path is updated in real time to avoid walking into or through other agents within the simulation. In order to determine this goal position, each 3D character was programmed to follow a simple logic tree and make decisions depending on their current situation. For example, an agent who has walked into the train carriage will 'look' for a free seat (deciding on the closest free seat near them) and then walk towards that seat. As it is walking the agent is constantly rechecking if the seat is still free (another agent could have taken it). If the seat is free and they are close enough to sit in it, they will sit down. Once the agent is seated, the simulation considers them 'complete' and no further action is required. A flowchart outlining the decisions that each agent makes can be seen below in Fig. 6.

\section{Application of this Technique with an Alternative Design of Carriage}

To test the efficacy of the modelling simulation, example carriage interior designs were prepared. This was part of a long-running larger project concerning dwell time and passenger dispersal behaviour. A model of the existing rolling stock was designed to create a baseline performance of the system. A concept design whereby the interior was re-arranged to accommodate a different seating arrangement and an alternative set of doors was devised. The alternative test design concept attempted to manipulate passenger flow with the following strategies:

- re-arranging seating into centrally mounted clusters to open dual corridors through the carriage; 
Fig. 4 A 3D character in 'walk cycle' and an idle loop for standing and sitting. Created by Chandara Ung
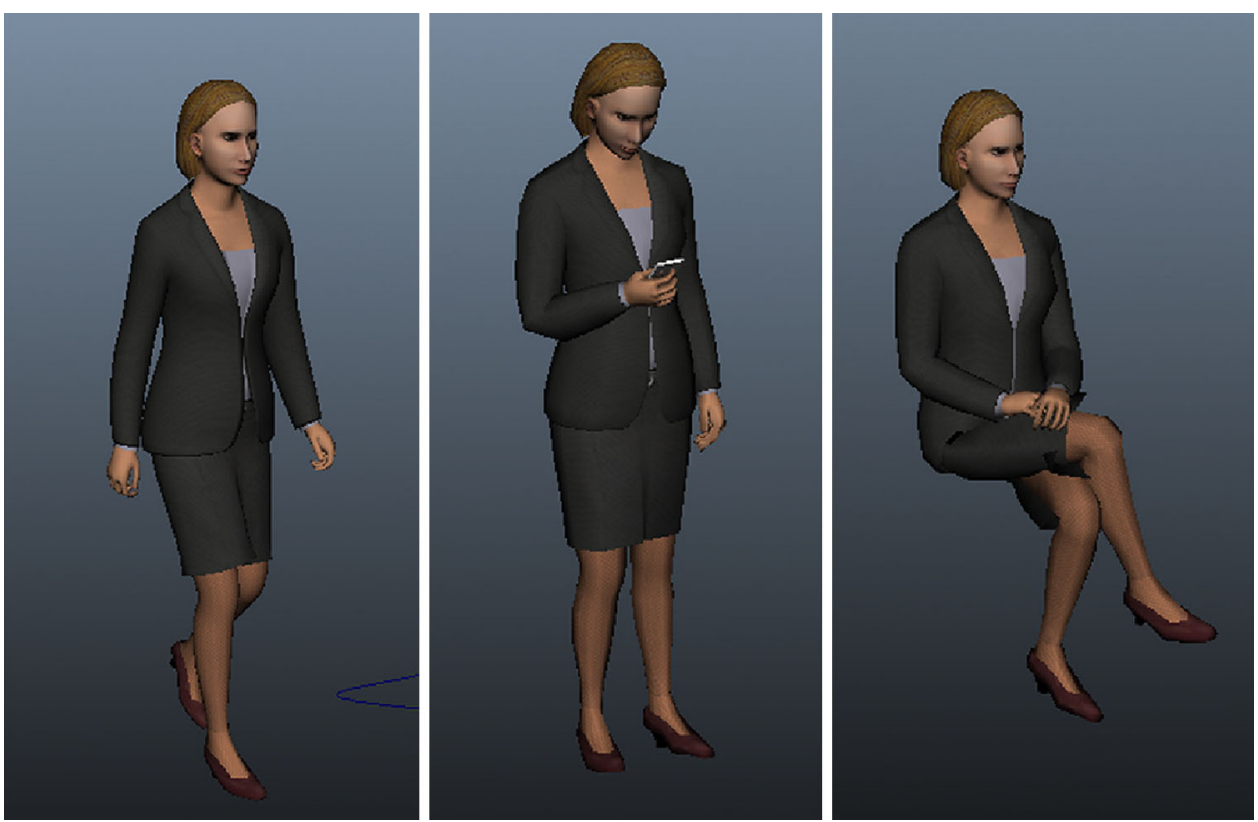

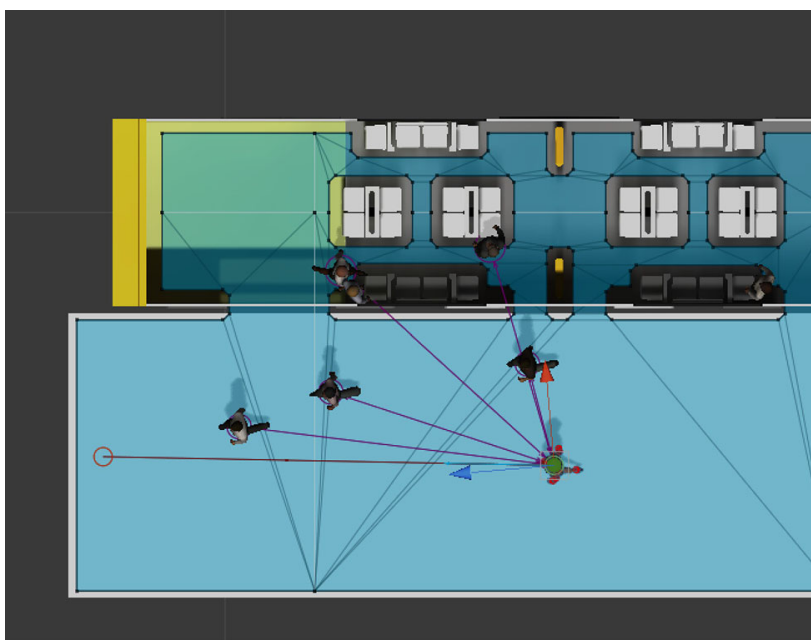

Fig. 5 Plan view of the mapping of agent movement in Unity. Areas permissible for agents to walk in are outlined in blue

- door arrangements that dictate passenger flow either by dint of their width (greater than $2 \mathrm{~m}$ ) or by the implementation of 'rules' to impose passenger behaviours, particularly in determining the direction of movement to and away from ingress and egress;

- creating the largest possible seating capacity.

The relative performances were then compared using the simulation with variable crowding figures. The conceptual design contained a central arrangement of seating clusters with dual corridors running along the length of the carriage and high numbers of folding and perch seats. The central innovation offered here is the concept of the 'peak door'. In essence, the authors are speculating that the three-door arrangement as utilised on contemporary trains remains in place and that extra boarding and alighting capacity is only required at certain times of day and at those times an extra two doors per side become operational. These peak doors would be relatively discreet during the off-peak period and indeed folding seats would be located across the temporary vestibule. These seats would fold into the door framework at designated times on the early shoulder of the peak period, locking into place as the doors become operative. Seating would be lost, but standing space is increased (Fig. 7).

The operational mechanism by which these peak doors are implemented would be aligned with the start and finish of services. A change in the prevailing culture of passenger expectation would be required for patrons to come to expect extra doors to be operative at certain times of the day. This concept opens up the notion of internal space being flexible beyond the use of folding seating. For most of the day, the services can manage a dispersed patronage through the carriage, and, with the exceptions of some accessibility issues, only at peak times do crowding, poor dispersal and lengthened dwell times reflect negatively on the carriage design. The use of peak doors, utilised only temporarily, overcomes the issue of the loss of seats due to multiple vestibule spaces.

\section{Results}

The simulation serves to validate, via experimentation, an improved boarding and alighting time. The validity of the conceptual carriage interior as an arbiter of improved passenger exchange and stabilised dwell times is seen most keenly by applying the simulation to the highest loads. 


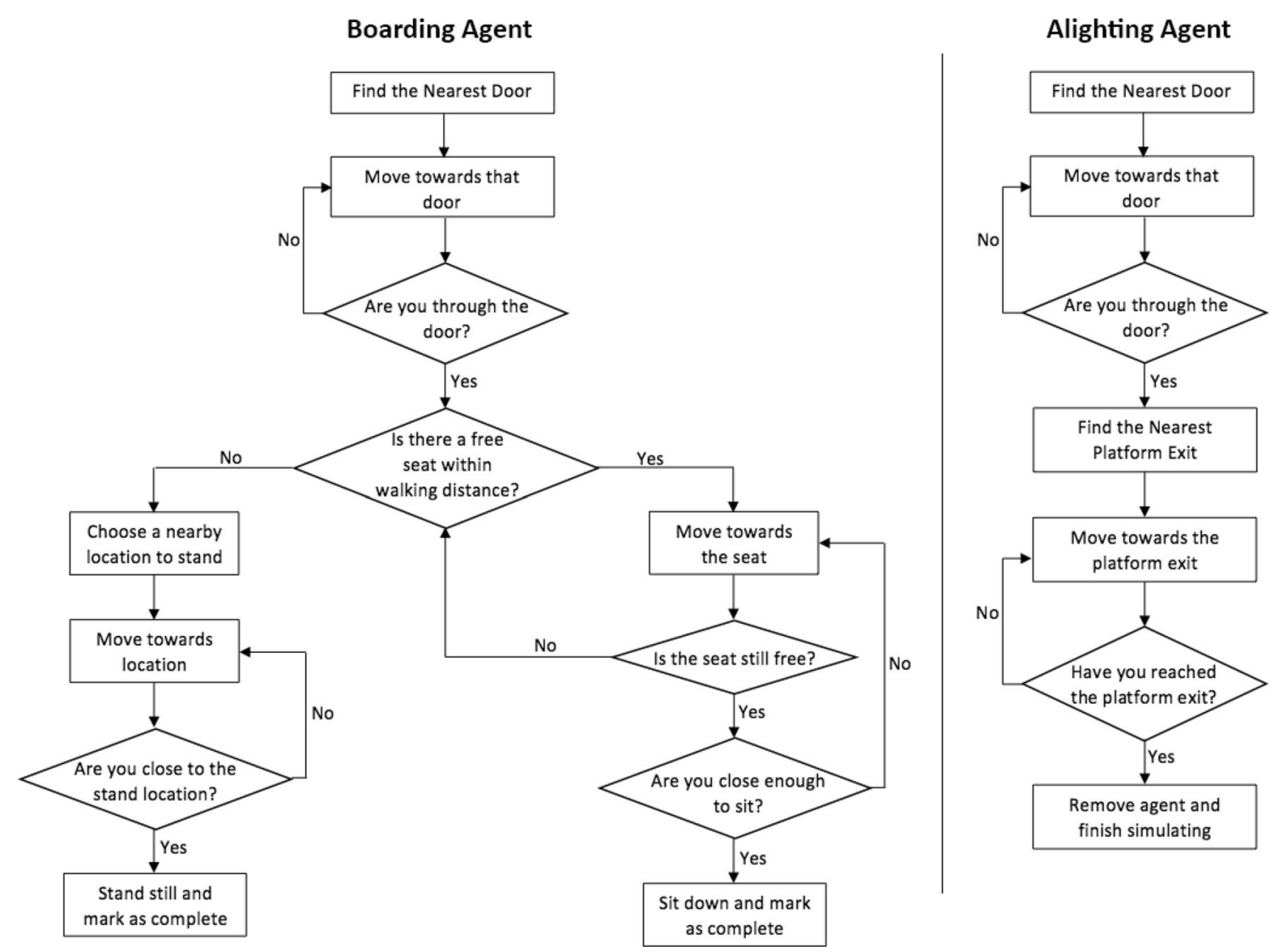

Fig. 6 Flowchart decision tree for agents moving through the simulation

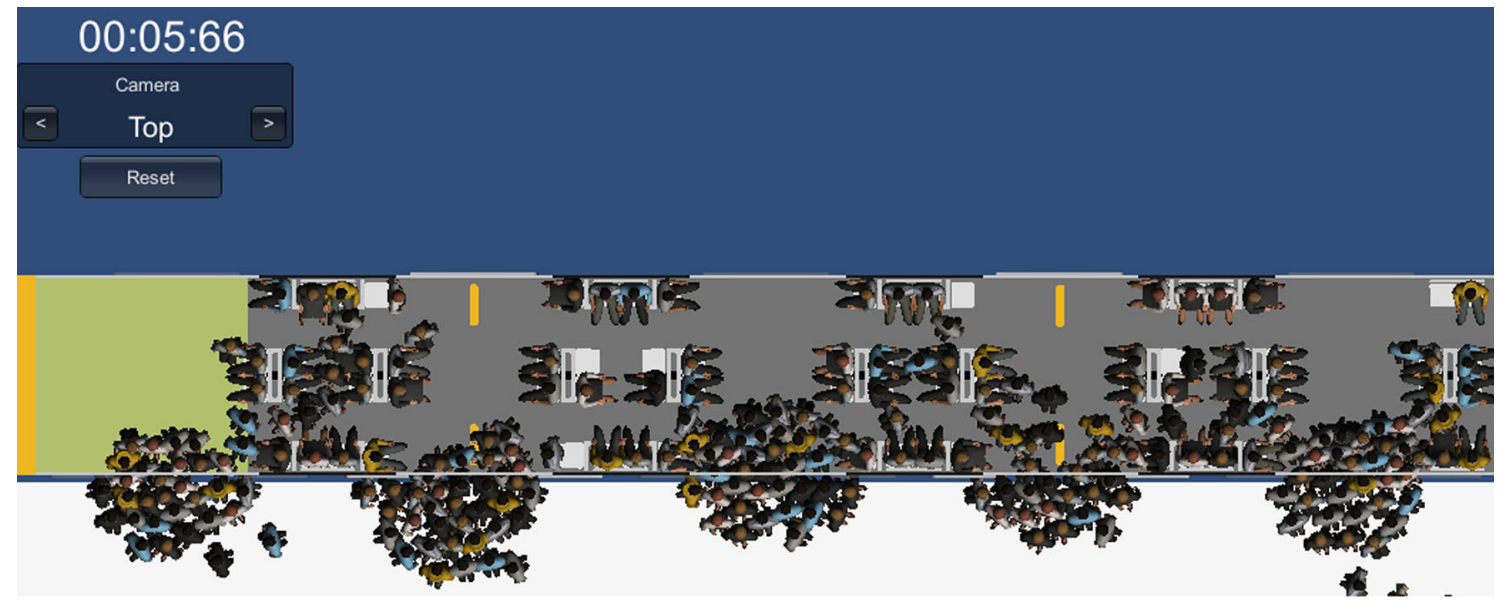

Fig. 7 Plan view of the simulation as applied to the concept interior where Peak Doors have been applied and there is a heavy patronage

The worst case situation for dwell time delays is caused when all the seats in the carriage are taken and the excess in capacity is beginning to build as standees cluster around the door vestibules. When such a train arrives at a significant interchange, where passengers need to alight and significant numbers need to board, then delays in dwell time occur.
In these simulations, both the existing rolling stock (Comeng) and the new concept carriage are populated to the capacity of the simulation with 250 passengers seated and standing. There are no datasets indicating the exact numbers of passenger exchanges, boarding, alighting and standees, so a number of simulations were created, incrementally increasing the percentage of passengers alighting 
and boarding. The percentage increments are based upon an existing study originally contracted by the Melbourne Transport Operating Company, in which Weston's formula was used to calculate a graphical distribution of anticipated dwell times. In each simulation, the number of passengers either boarding or alighting is increased by $5 \%$. The distribution of these passengers, both within the carriage and on the platform, is randomly driven by the Unity software and so no two simulations can be exactly repeated. For each of the conditions, both carriages fall within the generally accepted dwell time of $20 \mathrm{~s}$; however, the peak door two-corridor solution (Fig. 7) gives consistently quicker passenger exchanges. Only for very small numbers $(5 \%$ equating to 14 patrons) were exchange times roughly comparable. As the number of passengers in the boarding and alighting exchange increased, the extra door and corridor space had an observable impact on dwell time reduction.

As an example of an extreme exchange, simulations were run based on the very high passenger loads, with $36 \%$ above the intended carriage capacity. This figure is based on the recorded loading counts at Clifton Hill, Melbourne during May 2011. In this scenario, the train was carrying an average, assuming an even distribution, of 182 passengers per carriage. The test aimed to determine, at this high loading, the results if all the seats remained occupied and the remaining standing passengers all alighted from the carriage to be replaced by the same number boarding. Over 100 repeated simulations, on average the concept train carriage had a $33 \%$ shorter dwell time, although it was noted that neither the existing train design nor the concept achieved the desirable standard 20 s dwell time at these very high loads (Fig. 8).

\section{Future Work}

These initial simulations are encouraging in terms of the data they are able to generate and the insights gained therein to how passenger crowds might board, alight and disperse in various carriage arrangements. Extending the range of behaviours that $3 \mathrm{D}$ agents exhibit when reacting to other agents poses a number of interesting possibilities. If a crowd consists of passengers mostly travelling alone, the simulation could account for people's general preference in not only their spatial separation but also for seats facing forward and next to a window. The Authors' current simulation reflects an environment most like a peak time commute, whereby most passengers are not associated with a group of other people. What if our agents were travelling in the company of friends and companions? Fine tuning the simulation to account for groups of agents gathering together near the doors or in adjacent seats would entail a consideration for how other agents navigate around these groups. A large crowd of vocal high school students boarding at a station, for example, brings about a new range of agent behaviours and movement dynamics. We discussed above the possibility of agents moving at very different speeds, but how might agents be convincingly programmed to consider other agents, for example, to give up their seat or make way for elderly or disabled passengers? And finally, how might other agents react to groups of disruptive passengers or even aggressive and intoxicated ones? Refinements of agent behaviours such as these would clearly benefit from the collaboration of behavioural psychologists.

\section{Conclusion}

Commuter rail is experiencing growth in patronage with higher passenger densities and the effects of crowding, accessibility and extended dwell times. Research indicates that the design of the train carriage and the impediments of platform furniture all have an influence on dwell time performance and therefore network capacity. Building new train and platform infrastructure concepts are expensive undertakings and carry a high level of risk. The Authors have demonstrated that contemporary high-level game engine software can create authentic simulations of crowd behaviour and dispersal to the extent that designs can be tested in advance of implementation.

In these final simulations, it can be seen that multiple doors, dual-flow passenger exchange and dual corridors made for consistently shorter dwell times for the same numbers of patrons as in the current existing rolling stock. Certain assumptions have been made. Motivations for agents to seek a seat and obey certain cultural norms have been assumed and while observable evidence would suggest these assumptions have validity [3], this does not negate all possible behaviours that might be encountered at stations. Equally, passenger motivations discussed in the literature but not applied in these simulations due to technical difficulty include sitting next to known people or away from strangers, sitting in the direction of travel and next to windows, bunching at certain doors, as in rainy or hot sunny conditions and when some patrons circumnavigate control conventions and so work against prevailing crowd movements. Contemporary software such as the one used by the Authors enables these refinements to be undertaken in the next iteration of this simulation tool. 

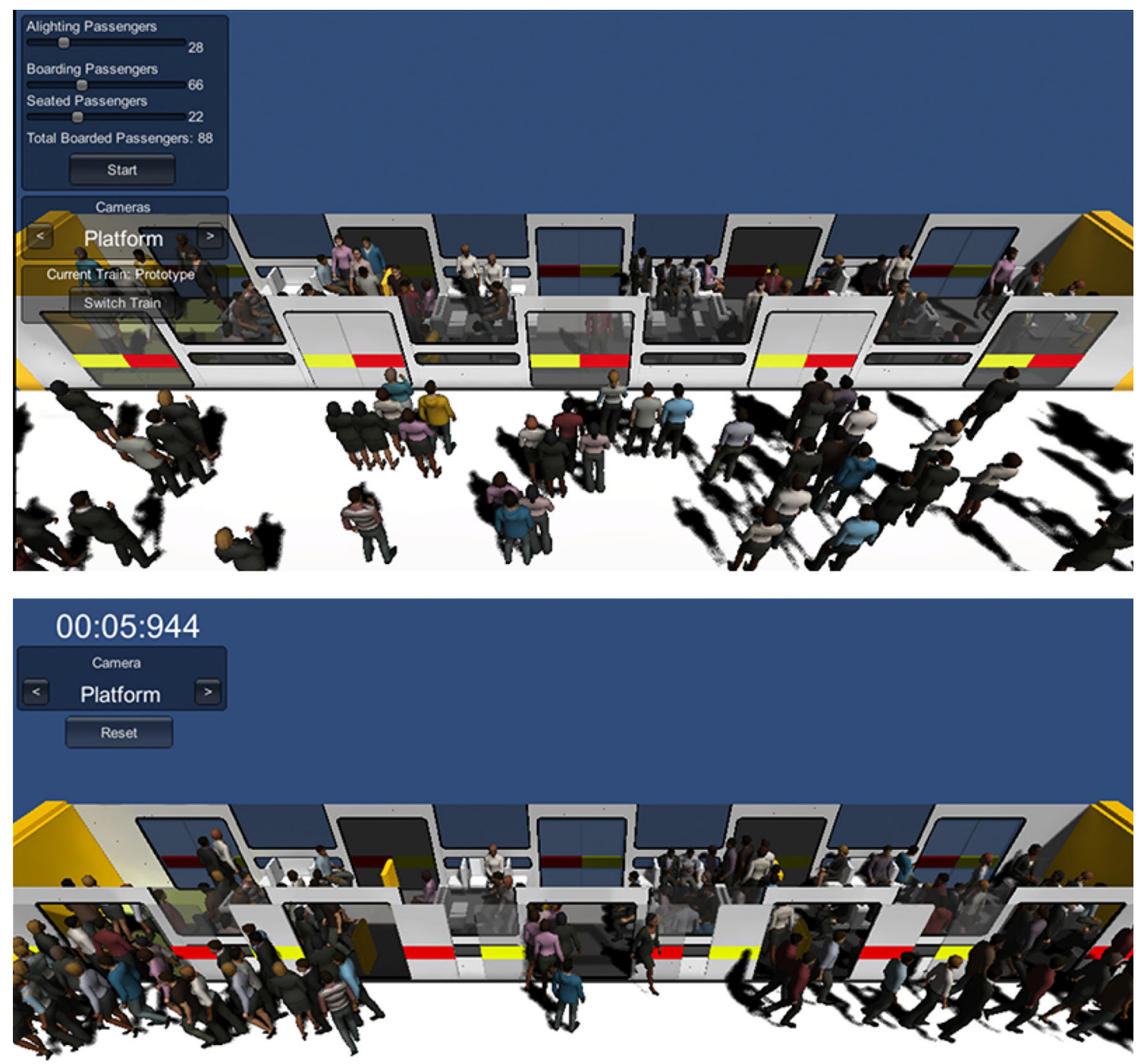

Fig. 8 A perspective view of the simulation demonstrating a realistic three-dimensional view of the passengers boarding and alighting. Note also the user interface in the top left corner of the screen where loading patterns of passengers is determined

Open Access This article is distributed under the terms of the Creative Commons Attribution 4.0 International License (http://crea tivecommons.org/licenses/by/4.0/), which permits unrestricted use, distribution, and reproduction in any medium, provided you give appropriate credit to the original author(s) and the source, provide a link to the Creative Commons license, and indicate if changes were made.

\section{References}

1. Burdett R, Sudjic D (2009) The endless city-the urban age project, London School of Economics \& Herrhausen Society. Phaidon, London
2. Daamen W, Lee Y, Wiggenraad P (2008) Boarding and alighting behaviour of public transport passengers. J Trans Res Board 2042:71-81. doi:10.3141/2042-08

3. Hirsch L, Thompson K (2011) I can sit but I'd rather stand: Commuter's experience of crowdedness and fellow passenger behaviour in carriages on Australian metropolitan trains. Australian Transport Research Forum 2011 Proceedings, Adelaide, Australia, 28-30 Sept 2011

4. Ruger B, Tuna D (2008) Influence of railway interiors on dwell time and punctuality. Railway Interiors International, UKIP Media Events, Dorking, Surrey

5. Wiggenraad P (2001) Alighting and boarding times of passengers at Dutch railway stations; analysis of data collected at seven stations in October 2000. TRAIL Research School. Delft University of Technology, Delft 